DOI: http://dx.doi.org/10.15688/jvolsu10.2014.6.10

УДК 536.7-631.152

ББК 65.01

\title{
ЭНТРОПИЙНАЯ НОМОГРАММА
}

\section{Кораблев Григорий Андреевич}

Профессор, доктор химических наук, заведующий кафедрой физики,

Ижевская государственная сельскохозяйственная академия korablevga@mail.ru ул. Студенческая, 11, 426069 г. Ижевск, Российская Федерация

\section{Петрова Наталья Григорьевна}

Специалист-эксперт отдела информационной безопасности и связи, Министерство информатизации и связи Удмуртской Республики biakaa@mail.ru ул. Вадима Сивкова, 186, 426057 г. Ижевск, Российская Федерация

\section{Кодолов Владимир Иванович}

Доктор химических наук, профессор, заведующий кафедрой химии и химической технологии, Ижевский государственный технический университет kodol@istu.ru ул. Студенческая, 7, 426000 г. Ижевск, Российская Федерация

\section{Заиков Геннадий Ефремович}

Доктор химических наук, профессор, заведующий отделом биологической и химической физики полимеров, Институт биохимической физики им. Н. М. Эмануэля РАН chembio@sky.chph.ras.ru ул. Косыгина, 4, 119334 г. Москва, Российская Федерация

\section{Кораблев Роман Григорьевич}

Аспирант,

Ижевская государственная сельскохозяйственная академия

korablevga@mail.ru

ул. Студенческая, 11, 426069 г. Ижевск, Российская Федерация 


\section{Осипов Анатолий Константинович}

Доктор экономических наук, профессор,

заведующий кафедрой менеджмента и права,

Ижевская государственная сельскохозяйственная академия

korablevga@mail.ru

ул. Студенческая, 11, 426069 г. Ижевск, Российская Федерация

Аннотация. Аналогично представлениям термодинамики о статистической энтропии использовано понятие энтропии пространственно-энергетических взаимодействий.

Получена номограмма для оценки энтропии различных процессов.

Обсуждается многоплановость проявлений энтропии, в том числе - в биохимических процессах и в экономике.

Ключевые слова: энтропия, номограмма, пространственно-энергетический параметр, карбонизация, диффузия, бизнес.

\section{1. Введение}

Понятие энтропии возникло на основе второго закона термодинамики и представлений о приведенном количестве теплоты.

В статистической термодинамике энтропия изолированной и находящейся в равновесии системы равна логарифму вероятности нахождения ее в определенном макросостоянии:

$$
S=k \ln W,
$$

где $W$ - число доступных состояний системы или степень вырождения микросостояний; $k$ - постоянная Больцмана.

Или:

$$
W=e^{s / k} .
$$

Эти соотношения являются общими утверждениями, имеющими макроскопический характер, не содержат никаких ссылок на элементы структур рассматриваемых систем и полностью не зависят от микроскопических моделей [2].

Поэтому применение и рассмотрение этих законов может иметь большое число следствий, которые наиболее плодотворно используются статистической термодинамикой.

Смысл второго закона термодинамики сводится к следующему.

Природа стремится от состояний менее вероятных к состояниям более вероятным. Так, наиболее вероятным является равномерное распределение молекул по всему объе- му. С макрофизической точки зрения эти процессы заключаются в выравнивании плотности, температуры, давления и химических потенциалов, а основной характеристикой процесса является термодинамическая вероятность $-W$.

В реальных процессах в изолированной системе рост энтропии неизбежен - в системе нарастает беспорядок, хаос, идет понижение качества внутренней энергии.

Термодинамическая вероятность равна числу микросостояний, отвечающих данному макросостоянию.

Поскольку степень вырождения системы никак не связана с физическими особенностями систем, статистическое понятие энтропии может иметь и другие применения и проявления (кроме статистической термодинамики).

«Ясно, что из двух совершенно разных по своему физическому содержанию систем энтропия может быть одинаковой, если у них число возможных микросостояний, отвечающих одному макропараметру (неважно, какой это параметр) совпадают. Именно поэтому понятие энтропии можно использовать в самых разнообразных областях. Возрастающая самоорганизация человеческого общества... приводит к возрастанию энтропии и беспорядка в окружающей среде, что выражается в частности в появлении громадного числа рассеянных по Земле свалок» [3].

В данном исследовании делается попытка применения понятия энтропии к оценке сте- 


\section{ТЕХНИКО-ТЕХНОЛОГИЧЕСКИЕ ИННОВАЦИИ}

пени пространственно-энергетических взаимодействий с использованием их графической зависимости и в других областях.

\section{2. Энтропийная номограмма степени пространственно-энергетических взаимодействий}

На основе модифицированного уравнения Лагранжа для относительного движения двух взаимодействующих материальных точек было введено представление о пространственно энергетическом параметре ( $P$-параметре), который является комплексной характеристикой важнейших атомных величин, ответственной за межатомные взаимодействия и имеющей прямую связь с электронной плотностью в атоме (см. рис. 1) [1].

В качестве основной количественной характеристики структурных взаимодействий в конденсированных средах использовалась величина относительной разности $P$ параметров взаимодействующих атомовкомпонентов - коэффициент $\alpha$ структурного взаимодействия:

$$
\alpha=\frac{P_{1}-P_{2}}{\left(P_{1}+P_{2}\right)-2} 100 \% .
$$

$\mathrm{C}$ применением надежных экспериментальных данных была получена номограмма зависимости степени структурных взаимодействий ( $\rho)$ от коэффициента $\alpha$, единая для широкого класса структур. Данный подход дал возможность оценить степень и направление структурных взаимодействий процессов фазообразования, изоморфизма и растворимос- ти в многочисленных системах, в том числе в молекулярных.

Такая номограмма может быть представлена [3] и в виде логарифмической зависимости:

$$
\alpha=\beta(\ln \rho)^{-1},
$$

где коэффициент $\beta$ - постоянная величина для данного класса структур. От среднего значения величина $\beta$ структурно может изменяться в основном только в пределах $\pm 5 \%$. Таким образом, величина $\alpha$ обратно пропорциональна логарифму степени структурных взаимодействий и поэтому может характеризоваться как энтропия пространственноэнергетических взаимодействий атомно-молекулярных структур.

Действительно, чем больше $\rho$, тем более вероятно образование стабильных упорядоченных структур (например, образование твердых растворов), то есть тем меньше энтропия процесса. Но тем меньше и коэффициент $\alpha$.

Уравнение (4) не имеет полной аналогии с уравнением (1) Больцмана, так как в данном случае сравниваются не абсолютные, а только относительные значения соответствующих характеристик взаимодействующих структур, которые могут выражаться в процентах. И это касается не только коэффициента $\alpha$, но и сравнительной оценки степени структурных взаимодействий ( $\rho)$, например - процент содержания атомов данного элемента в твердом растворе относительно общего числа атомов.

Поэтому в уравнении (4) коэффициент $\beta=1$.

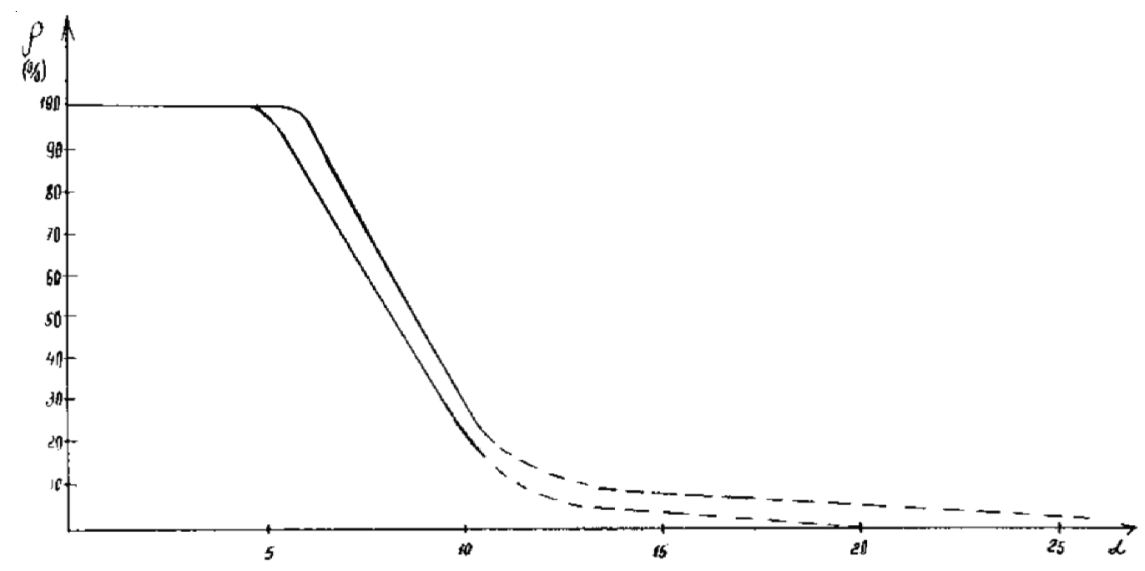

Рис. 1. Номограмма зависимости степени структурных взаимодействий ( $\rho)$ от коэффициента $\alpha$ 


\section{ТЕХНИКО-ТЕХНОЛОГИЧЕСКИЕ ИННОВАЦИИ}

Вывод: относительная разность пространственно-энергетических параметров взаимодействующих структур может быть количественной характеристикой энтропии взаимодействия: $\alpha \equiv S$.

\section{3. Энтропийная номограмма поверхностно-диффузионных процессов}

В качестве примера рассмотрим процесс карбонизации и формирования наноструктур при взаимодействиях в гелях поливинилового спирта и металлической фазы в виде оксидов или хлоридов меди. На первом этапе образуются малые кластеры неорганической фазы, окруженные углеродосодержащей фазой. В этот период основной характер атомно-молекулярных взаимодействий должен оцениваться через относительную разность $P$-параметров, рассчитанных через радиусы ионов меди и ковалентные радиусы атомов углерода, приведенные в таблице 1.

Из таблицы 1 видно, что в этом случае коэффициент $\alpha=3,50$, что соответствует полному структурному взаимодействию: $\rho=100 \%$. При этом процесс происходит только в объеме геля, а не на поверхности несформировавшейся пока пленки.

В следующем основном периоде карбонизации идет образование металлической фазы непосредственно на поверхности образующихся полимерных структур по реакции:

$$
2 \mathrm{CuCl}+[-\mathrm{CH}=\mathrm{CH}-]_{n} \rightarrow 2 \mathrm{Cu}+2 \mathrm{HCl}+\left[\mathrm{C}_{2}\right]_{n} .
$$

С этого времени начинается процесс формирования бинарной матрицы наносистемы $\mathrm{C} \rightarrow \mathrm{Cu}$. Рассмотрим ход нарастания пленочной матрицы углеродов в меди в модели поверхностной диффузии. В этот период образования металлической фазы валентно активными являются $P$-параметры, рассчитанные через радиусы атомов.

В жидкости радиус сферы молекулярного взаимодействия $\mathrm{RH} \approx 3 r$, где $r$ - радиус молекулы. Жидкости образуются главным образом элементами первого и второго периодов системы. Для второго периода можно записать: $\mathrm{RH} \approx 3 r=(n+1) r$, где $n-$ главное квантовое число. Для обоих периодов (первого и второго) получаем $\mathrm{R}=(<n>+1) r \approx 2,5 r$.

Предположим, что этот принцип с определенным приближением можно распространить на различные элементы всех остальных периодов, но с учетом экранирующих эффектов, вводя вместо $n$ величину эффективного главного квантового числа $\left(n^{*}\right)$. Эти значения $n^{*}$ и $n^{*}+1$, взятые по Слэтеру [5], представлены в таблице 2.

Итак, предполагаем, что радиус сферы атомно-молекулярного взаимодействия при диффузии частиц определяется так:

$$
R=\left(n^{*}+1\right) r,
$$

где $r$ - размерная характеристика атомной структуры. Общее изменение $R$ составляет от $3 r$ до $5,2 r$ (со второго по шестой периоды).

\begin{tabular}{|c|c|c|c|c|c|c|c|c|c|c|c|c|}
\hline \multicolumn{4}{|c|}{ Атом углерода } & \multicolumn{3}{|c|}{ Атом меди } & \multicolumn{6}{|c|}{ Характеристики взаимодействий } \\
\hline $\begin{array}{l}\text { Орби- } \\
\text { таль }\end{array}$ & \begin{tabular}{|c|}
$\frac{P_{o}}{R\left(n^{*}+1\right)}$ \\
$(\ni \mathrm{B})$
\end{tabular} & $\begin{array}{c}\frac{P_{o}}{r_{u}\left(n^{n}+1\right)} \\
(\mathrm{BB})\end{array}$ & K & $\begin{array}{l}\text { Орби- } \\
\text { таль }\end{array}$ & 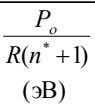 & $\begin{array}{c}\frac{P_{o}}{r_{u}\left(n^{*}+1\right)} \\
(\exists \mathrm{B})\end{array}$ & $\begin{array}{c}\alpha \\
(\%)\end{array}$ & $\begin{array}{l}1 / \alpha \\
(\%)\end{array}$ & $\begin{array}{c}\rho \\
(\%)\end{array}$ & $\begin{array}{c}t \\
\text { (ч) }\end{array}$ & $\begin{array}{c}\omega \\
(\%)\end{array}$ & $\begin{array}{c}\text { Вид } \\
\text { взаимодей- } \\
\text { ствий }\end{array}$ \\
\hline $2 \mathrm{P}^{2} 2 \mathrm{~S}^{2}$ & & 3,1519 & 1 & $4 \mathrm{~S}^{2}$ & & 3,0436 & 3,50 & 0,29 & 100 & 0 & 0 & объемная \\
\hline $2 \mathrm{P}^{2}$ & 4,3554 & & 1,6 & $4 S^{1} 3 d^{1}$ & 2,2011 & & 21,17 & 0,05 & $5-8$ & 0 & 0 & $\begin{array}{c}\text { полу- } \\
\text { поверхно- } \\
\text { стная }\end{array}$ \\
\hline $2 \mathrm{P}^{2}$ & 4,3554 & & 1,7 & $4 S^{1} 3 d^{1}$ & 2,2011 & & 15,15 & 0,07 & $19-21$ & 0,49 & 21,5 & $\begin{array}{c}\text { поверхно- } \\
\text { стная }\end{array}$ \\
\hline $2 \mathrm{P}^{2}$ & 4,3554 & & 1,8 & $4 S^{1} 3 d^{1}$ & 2,2011 & & 9,46 & 0,11 & $56-58$ & 1,05 & 63,9 & $\begin{array}{l}\text { поверхно- } \\
\text { стная }\end{array}$ \\
\hline $2 \mathrm{P}^{2}$ & 4,3554 & & 1,9 & $4 S^{1} 3 d^{1}$ & 2,2011 & & 4,06 & 0,25 & $\sim 98-100$ & 1,6 & 95 & $\begin{array}{c}\text { поверхно- } \\
\text { стная }\end{array}$ \\
\hline $2 \mathrm{P}^{2}$ & 4,3554 & & 2,0 & $4 S^{1} 3 d^{1}$ & 2,2011 & & 1,07 & 0,93 & 100 & 2,0 & 98,3 & $\begin{array}{c}\text { поверхно- } \\
\text { стная }\end{array}$ \\
\hline
\end{tabular}

Структурные взаимодействия при образовании нанопленки в системе $\mathrm{C} \rightarrow \mathbf{C u}$ 
Таблица 2

Эффективное главное квантовое число

\begin{tabular}{|c|c|c|c|c|c|c|}
\hline$n$ & 1 & 2 & 3 & 4 & 5 & 6 \\
\hline$n^{*}$ & 1 & 2 & 3 & 3,7 & 4 & 4,2 \\
\hline$n^{*}+1$ & 2 & 3 & 4 & 4,7 & 5 & 5,2 \\
\hline
\end{tabular}

Усредненное значение структурного $P_{c}$ параметра, приходящегося на единицу радиуса атомно-молекулярного взаимодействия, определяется по уравнению:

$$
P_{c}=\frac{P_{\mathrm{o}}}{K R}=\frac{P_{0}}{r\left(n^{*}+1\right) K},
$$

где $K$ - коэффициент, учитывающий относительное число взаимодействующих частиц и равный (как показали расчеты):

$$
K=N_{0} / N^{*}
$$

Здесь $N_{0}$ - число частиц в объеме сферы радиуса $R, N$ - число частиц или реализованных взаимодействий в зависимости от вида процесса (внутренней или поверхностной диффузии).

Внутри жидкости, ниже верхнего слоя толщиной $2 R$ равнодействующая сил молекулярного взаимодействия равна нулю (см. рис. 2).

Применяя исходную аналогию к внутренней диффузии, можно считать, что такое равновесное состояние соответствует равенству $N_{0}=N$, и тогда $K=1$.

На верхней части поверхностного слоя жидкости объем сферы атомно-молекулярного взаимодействия и число частиц в ней практически в 2 раза меньше по сравнению с внутренними слоями ниже $2 R$, то есть $\frac{N_{0}}{N} \approx 2$ и $K=2$ для поверхностной диффузии (см. рис. 2).
Практически поверхностная диффузия идет при изменении коэффициента $K$ в пределах от 1,5 до 2,0 , что учтено в расчетах. На основе таких исходных положений был проведен расчет величины $P$-параметра и коэффициента $1 / \alpha_{2}$ по уравнениям (3), (6), (7) для атома углерода и меди (см. табл. 1).

Были вычислены значения степени структурных взаимодействий от коэффициента $\alpha$, то есть $\rho_{2}=f\left(1 / \alpha_{2}\right)$ - кривая 2 , представленная на рисунке 3. Здесь приведена также графическая зависимость степени образования нанопленок $(\omega)$ от времени процесса по данным работы [6] - кривая 1 и ранее полученная номограмма энтропии в виде $\rho_{1}=f\left(1 / \alpha_{1}\right)-$ кривая 3 .

Анализ всех полученных графических зависимостей показывает почти полное графическое совпадение всех трех графиков: $\omega=f(t), \rho_{1}=f\left(1 / \alpha_{1}\right), \rho_{2}=f\left(1 / \alpha_{2}\right)-$ с небольшими отклонениями в начале и в конце процесса. Таким образом, скорость карбонизации, как и функции многих других физико-химических структурных взаимодействий, могут непосредственно оцениваться через значения рассчитанных величин коэффициента $\alpha$ и энтропийную номограмму.

\section{4.Номограммы биофизических процессов}

1) О кинетике ферментативных процессов. «Необходимым этапом ферментативного катализа является образование фермент-

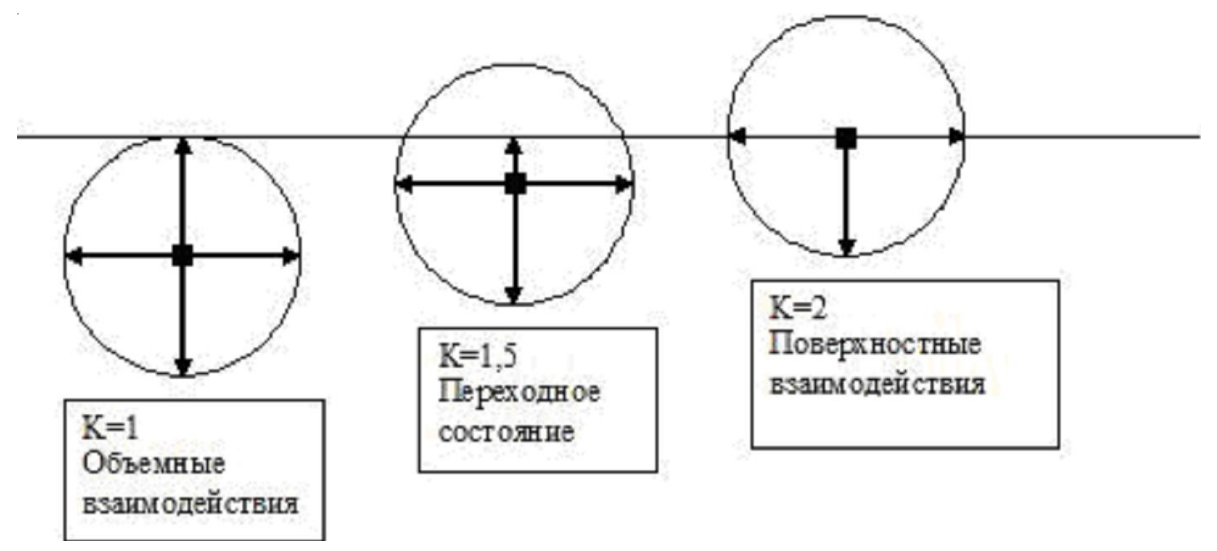

Рис. 2. Относительное число взаимодействующих частиц 


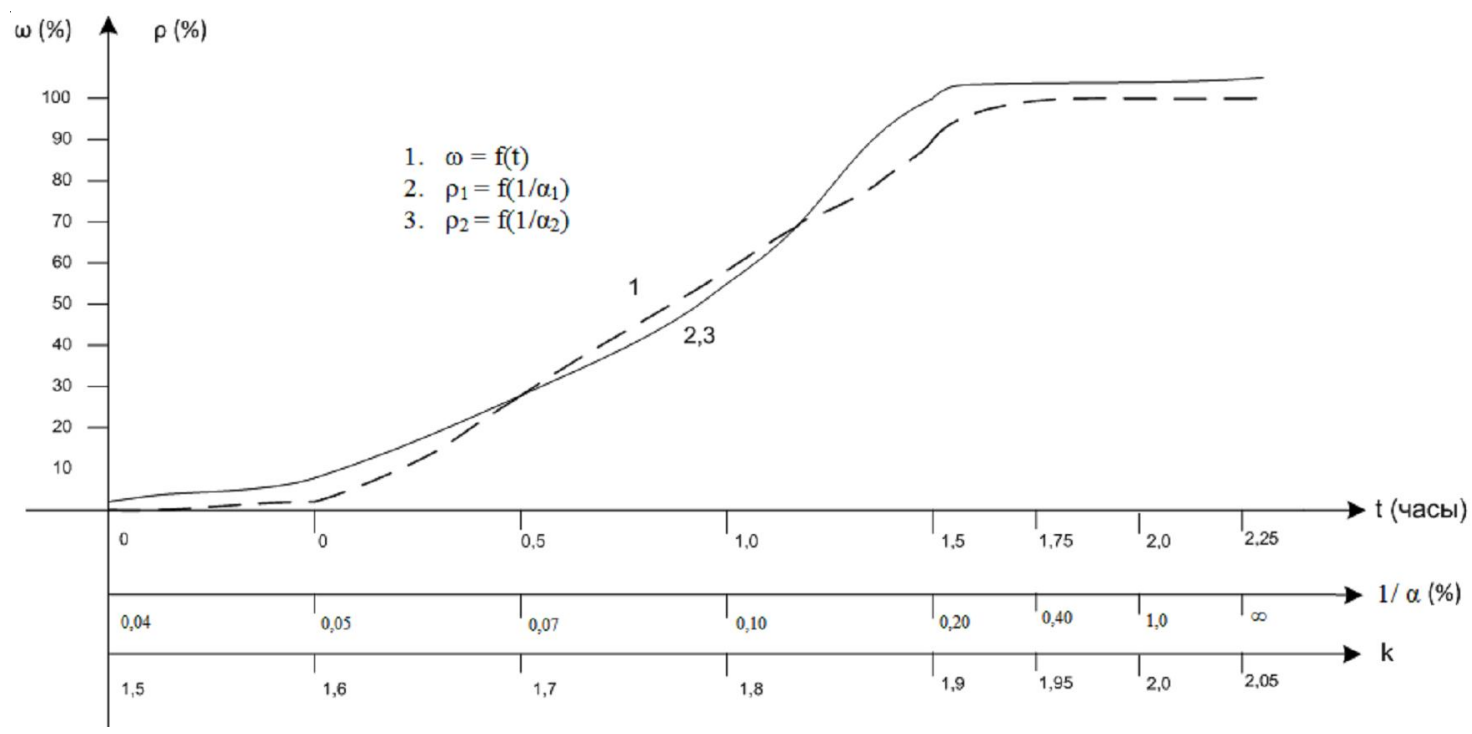

Рис. 3. Зависимость скорости карбонизации от коэффициента $\alpha$

субстратного комплекса... При этом, к молекуле фермента может присоединиться $\mathrm{n}$ молекул субстрата» [7, с. 58].

Для ферментов со стехиометрическим коэффициентом $n$, отличным от единицы, вид графической зависимости скорости наработêè ï đî äóêòà đåàêöèè $(\mu)$ в зависимости от концентрации субстрата (c) имеет [7] сигмоидный характер с характерной точкой перегиба (см. рис. 4).

Из рисунка 4 видно, что эта кривая повторяет в целом характер энтропийной номограммы на рисунке 3.

Аналогичный вид имеет график зависимости скорости электронного транспорта в биоструктурах от времени диффузии ионов [7, c. 278].

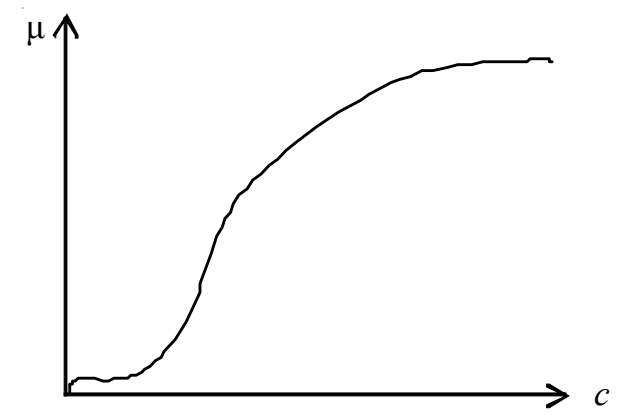

Рис. 4. Зависимость скорости ферментативной реакции $(\mu)$ от концентрации субстрата $(c)$

И в методике оценки ферментативных взаимодействий (аналогично ранее использо- ванной в подразделе 3 методике для поверхностно-диффузионных процессов) применяется эффективное число взаимодействующих молекул, которое больше 1.

В методологии $P$-параметра фермент имеет ограниченное изоморфное сходство с молекулами субстрата и не образует с ним стабильного соединения, но при этом возможна такая ограниченная перестройка химических связей, которая будет «настроена» на получение конечного продукта.

2) Зависимость биофизических критериев от их частотных характеристик.

a) Прохождение переменного тока через живые ткани характеризуется дисперсионной кривой электропроводности - это графическая зависимость полного сопротивления ткани (z-импеданс) от логарифма частоты переменного тока $(\log \omega)$. В норме такая кривая, на которой по оси ординат откладывается импеданс, а по оси абсцисс - $\log \omega$, формально полностью соответствует энтропийной номограмме (см. рис. 1).

б) Флуктуации проводимости биомембран (обусловленные случайными процессами) «имеют вид кривой Лоренца» $[8$, с. 99]. В этом графике спектральная плотность флуктуаций ( $\rho)$ откладывается на оси ординат, а функция логарифма частоты $(\log \omega)$ на оси абсцисс.

Вид такой кривой также соответствует энтропийной номограмме на рисунке 1. 


\section{5. Кривая Лоренца \\ пространственно-временной зависимости}

Интервалы между событиями в различных системах координат определяются лоренцовой геометрией пространства времени. В этой геометрии скорость ( $\beta)$ сама по себе не аддитивна, поэтому вводится понятие параметра скорости $(\theta)$. Связь между скоростью $\beta$ и параметром скорости простая: $\beta=$ th $\Theta$, где величина th $\Theta$ означает «тангенс гиперболический», и закон сложения двух скоростей имеет вид:

$$
\operatorname{th} \Theta=\operatorname{th}\left(\Theta_{1}+\Theta_{2}\right)=\frac{\operatorname{th} \Theta_{1}+\operatorname{th} \Theta_{2}}{1+\operatorname{th} \Theta_{1} \operatorname{th} \Theta_{2}} .
$$

Зависимость между параметром скорости и самой скоростью отображается [4] кривой Лоренца (рис. 5). Обе величины используются в относительных единицах по отношению к скорости света. Вид кривой формально полностью соответствует энтропийной номограмме на рисунке 3 .

Пример: «Пусть пуля выпускается со скоростью $\beta^{\prime}=0,75$ из ракеты, движущейся со скоростью $\beta_{r}=0,75$. Требуется найти скорость пули $\beta$ относительно лабораторной системы. Мы знаем, что аддитивны не скорости, а параметры скорости. По графику для точки $A$ находим $\theta^{\prime}=\Theta_{r}=0,973$. Сложение дает $\theta=\theta^{\prime}+\theta_{r}=1,946$. Для этого значения параметра скорости находим по графику точку $B$ и величину скорости $\beta=0,96 »[4]$.

\section{6. Энтропийные критерии в бизнесе и в природе}

Основные свойства системы свободного рынка, обеспечивающие ее экономические преимущества, это: 1) эффективная конкуренция и 2) максимальная личная заинтересованность каждого сотрудника.

Но на различных уровнях концентрации экономики эти первопричинные особенности функционируют и проявляют себя по-разному. Наибольшая их эффективность соответствует малому бизнесу, когда число членов организации минимально, более четкая личная заинтересованность и активная конкурентная борьба за выживание. По мере укрупнения предприятий и производств, с увеличением численности персонала роль каждого из них постепенно снижается, уменьшается конкурентная борьба, так как появляются новые возможности для согласованных действий разных бизнес-структур. Идет снижение качества экономических отношений в бизнесе, то есть возрастание энтропии. Более всего такой процесс характерен для моноструктур на крупнейших предприятиях большого бизнеса (синдикаты и картели).

Понятие термодинамической вероятности как числа микросостояний, отвечающих данному макросостоянию, можно модифицировать применительно к процессам экономических взаимоотношений, которые напрямую зависят от параметров бизнес-структур.

За макросостояние системы можно принять данную отдельную бизнес-структу-

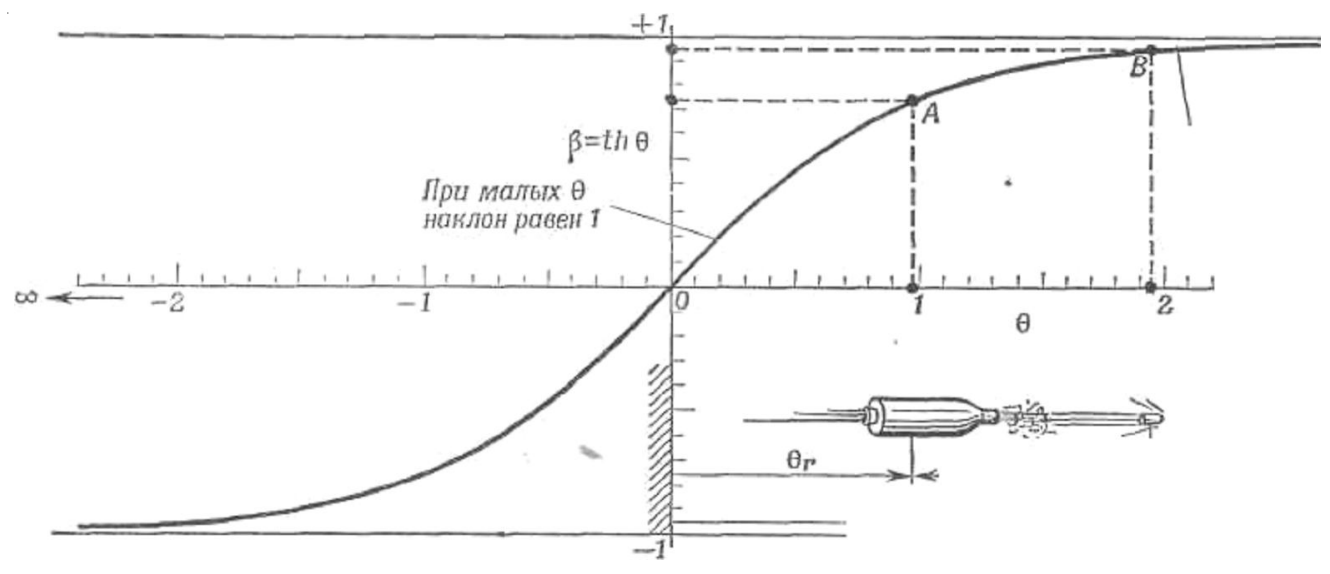

Рис. 5. Связь между параметром скорости и и самой скоростью, получаемая непосредственно из закона сложения 
py, a за число микросостояний - число ее сотрудников $(N)$, которое есть число доступных наиболее вероятных состояний данной бизнес-структуры. Таким образом, предполагается, что такое число сотрудников бизнес-структуры является аналогом термодинамической вероятности применительно к процессам экономических взаимоотношений в бизнесе.

Поэтому можно принять, что общая энтропия качества бизнеса состоит из двух энтропий, характеризующих: 1) уменьшение эффективности конкуренции $\left(S_{1}\right)$ и 2$)$ уменьшение личной заинтересованности каждого сотрудника $\left(S_{2}\right)$, то есть: $S=S_{1}+S_{2}$. Величина $S_{1}$ пропорциональна числу работников предприятия: $S \sim N$, а величина $S_{2}$ имеет сложную зависимость не только от числа работников предприятия, но и от эффективности самого управления им. Она обратно пропорциональна персональной заинтересованности каждого сотрудника. Поэтому можно принять, что $S_{2}=1 / \gamma$, где $\gamma$ - коэффициент личной заинтересованности каждого сотрудника.

По аналогии с уравнением Больцмана (1) получаем:

$$
\begin{gathered}
S=\left(S_{1}+S_{2}\right) \sim\left[\ln N+\ln \left(\frac{1}{\gamma}\right)\right] \sim \ln \left(\frac{N}{\gamma}\right) \\
S=k \ln \left(\frac{N}{\gamma}\right),
\end{gathered}
$$

или

где $k$ - коэффициент пропорциональности.

Здесь $N$ показывает, во сколько раз данная бизнес-структура больше эталонной структуры малого бизнеса, при которой $N=1$, то есть эта величина не имеет наименования.

Для нетермодинамических систем принимаем $k=1$. Поэтому

$$
S=\ln \left(\frac{N}{\gamma}\right) .
$$

В таблице 3 приведены примерные расчеты бизнес-энтропии по уравнению (8) для трех основных уровней бизнеса: малого, среднего и крупного. При этом предполагалось, что число $N$ соответствует некоторому среднему значению из наиболее вероятных величин.

При расчете коэффициента личной заинтересованности $\gamma$ учитывалось, что он может меняться от 1 (один сотрудник работает только сам на себя) до ноля (0), если такой работник - как бесправный раб, и для наиболее крупных предприятий принималось $\gamma=0,1-0,01$.

Несмотря на вполне приближенную точность таких усредненных расчетов, можно сделать достаточно достоверный вывод о том, что энтропия бизнеса с укрупнением его структур резко возрастает при переходе именно от среднего к крупному бизнесу, так как снижается качество бизнес-процессов.

В живых системах нарастание энтропии компенсируется через отрицательную энтропию (негоэнтропию), которая формируется через взаимодействие с внешней средой. То есть живая система - это открытая система. И бизнес не может длительное время быть изолированной системой, без процесса обмена и взаимоотношений с внешней средой. Роль внешней системы, уменьшающей возрастание бизнес-энтропии, должны выполнять, например, соответствующие государственные и общественные структуры, функционально отделенные от бизнеса. Возможно, неизбежным процессом в этом направлении может стать демонополизация крупнейших экономических структур, проводимая «сверху» эволюционным путем.

В термодинамике считается, что неконтролируемый рост энтропии ведет к прекращению всяких макроизменений в системах, то

Рост энтропии по мере укрупнения бизнеса

\begin{tabular}{|c|c|c|c|}
\hline \multirow{2}{*}{ Параметры структур } & \multicolumn{3}{|c|}{ Бизнес } \\
\cline { 2 - 4 } & Малый & Средний & Крупный \\
\hline$N_{1}-N_{2}$ & $10-50$ & $100-1000$ & $10000-100000$ \\
\hline$\gamma$ & $0,9-0,8$ & $0,6-0,4$ & $0,1-0,01$ \\
\hline $\mathrm{S}$ & $2,408-4,135$ & $5,116-7,824$ & $11,513-16,118$ \\
\hline$\langle\mathrm{S}\rangle$ & 3,271 & 6,470 & 13,816 \\
\hline
\end{tabular}




\section{ТЕХНИКО-ТЕХНОЛОГИЧЕСКИЕ ИННОВАЦИИ}

есть к их гибели. Поэтому актуальной является задача поисков методов снижения неконтролируемого роста энтропии в крупном бизнесе. При этом такие критические цифры энтропии относятся в основном именно к крупному бизнесу. Простое сокращение численности его сотрудников не может дать реального результата уменьшения энтропии. Так, сокращение числа работников на $10 \%$ дает уменьшение у них энтропии только на $0,6 \%$, и это при общих негативных явлениях безработицы, которая неизбежно сопровождает такой процесс.

Поэтому для таких сверхмоноструктур, не контролируемых ни государством, ни обществом, для уменьшения энтропии бизнеса более реален путь демонополизации без оптимизации (то есть без сокращения общего числа сотрудников).

Сравнение номограммы (см. рис. 1) с данными таблицы 3 показывает аддитивность численных величин энтропии бизнеса $(S)$ со значениями коэффициента пространственно-энергетических взаимодействий $(\alpha)$, то есть $S=\alpha$.

Поэтому применительно к бизнес-процессам понятие качества бизнеса аналогично понятию степени структурного взаимодействия $(\rho)$.

Все это позволяет приближенно определить критические значения данных величин. Так, при $\rho \approx 10 \%$ величина $S=\alpha \approx 12-18 \%$, что соответствует численности бизнес-структур в пределах от 10000 до 100000 сотрудников (в среднем около 55 000).

Оптимальные критерии более качественного бизнеса определяются максимальным значением их энтропии: $S=6-7$ (в относительных единицах).

Эти же значения ранее получены и для более полной степени структурных взаимодействий, так как непрерывные твердые растворы соответствуют значению.

Известно также, что максимально приемлемое для стабильной системы число атомов в полимерной цепи составляет около 100 единиц, что в кубическом объеме дает $10^{6}$. И тогда снова получаем $\lg 10^{6}=6$.

Сейчас научный мир озадачен интенсификацией технологических процессов на основе энергосберегающих электротехнологий (см., например, работы [9; 10]). Данная мето- дика $P$-параметра также может быть использована и в этом перспективном направлении.

\section{СПИСОК ЛИТЕРАТУРЫ}

1. Активность наноструктур и проявление ее в нанореакторах полимерных матриц и в активных средах / В. И. Кодолов, Н. В. Хохряков, В. В. Тринеева, И. И. Благодатских // Химическая физика и мезоскопия. -2008 . - Т. 10, № 4. - С. 448-460.

2. Бацанов, С. С. Интегралы перекрывания и проблема эффективных зарядов / С. С. Бацанов, Р. А. Звягина. - Новосибирск : Наука, Сиб. отд., 1966. $-386 \mathrm{c}$.

3. Грибов, Л. А. Основы физики / Л. А. Грибов, Н. И. Прокофьева. - М. : Высш. шк., 1992. - 430 с.

4. Новый подход в управлении утилизацией вторсырья в АПК / А. А. Смирнова, Н. А. Алексеева, И. Г. Поспелова, И. В. Возмищев // Всероссийская научно-практическая конференция «Инновации в науке, технике и технологиях», 28-30 апр. 2014 г. Ижевск : Удмуртский университет, 2014. - С. 249-250.

5. Поспелова, И. Г. Сублимационная сушка с комбинированным энергоподводом / И. Г. Поспелова, Я. Н. Захарова, Ф. В. Габасова // Механизация и электрификация сельского хозяйства. - 2009. № 6. - С. 30-32.

6. Рейф, Статистическая физика / Ф. Рейф. М. : Наука, 1972. -352 с.

7. Рубин, А. Б. Биофизика. Кн. 1. Теоретическая биофизика / А. Б. Рубин. - М. : Высш. шк., 1987. $-319 \mathrm{c}$.

8. Рубин, А. Б. Биофизика. Кн. 2. Биофизика клеточных процессов / А. Б. Рубин. - М. : Высш. шк., 1987. - $303 \mathrm{c}$.

9. Тейлор, Э. Физика пространства-времени / Э. Тейлор, Дж. Уилер. - М. : Мир, 1971. - 320 c.

10. Korablev, G. A. Spatial-Energy Principles of Complex Structures Formation / G. A. Korablev. Brill Academic Publishers and VSP, Netherlands, 2005. $-426 \mathrm{p}$.

\section{REFERENCES}

1. Kodolov V.I., Khokhryakov N.V., Trineeva V.V., Blagodatskikh I.I. Aktivnost nanostruktur i proyavlenie ee $\mathrm{v}$ nanoreaktorakh polimernykh matrits i $\mathrm{v}$ aktivnykh sredakh [Active Nanostructures and Their Manifestation in Nanoreactors of Polymer Matrix and Active Environments]. Khimicheskaya fizika i mezoskopiya, 2008, vol. 10, no. 4, pp. 448-460.

2. Batsanov S.S. Zvyagina R.A. Integraly perekryvaniya i problema effektivnykh zaryadov [The 


\section{ТЕХНИКО-ТЕХНОЛОГИЧЕСКИЕ ИННОВАЦИИ}

overlap Integrals and the Problem of Effective Charges]. Novosibirsk, Nauka Publ., 1966. 386 p.

3. Gribov L.A. Prokofyeva N.I. Osnovy fiziki [Fundamentals of Physics]. Moscow, Vysshaya shkola Publ., 1992. 430 p.

4. Smirnova A.A., Alekseeva N.A., Pospelova I.G., Vozmishchev I.V. Novyy podkhod v upravlenii utilizatsiey vtorsyrya v APK [A New Approach in the Management of the Disposal of Recyclable Materials in AIC]. Vserossiyskaya nauchno-prakticheskaya konferentsiya "Innovatsii $v$ nauke, tekhnike i tekhnologiyakh”, 28-30 aprelya 2014 g. [All-Russian Research and Practice Conference "Innovations in Science, Technique and Technologies", April 28-30, 2014]. Izhevsk, Izd-vo Udmurtskogo universiteta, 2014, pp. 249-250.

5. Pospelova I.G., Zakharova Ya.N., Gabasova F.V. Sublimatsionnaya sushka s kombinirovannym energopodvodom [Freeze Drying With a Combined
Power Supply]. Mekhanizatsiya i elektrifikatsiya selskogo khozyaystva, 2009, no. 6, pp. 30-32.

6. Reyf F. Statisticheskaya fizika [Statistical Physics]. Moscow, Nauka Publ., 1972. 352 p.

7. Rubin A.B. Biofizika. Kn.1. Teoreticheskaya biofizika [Biophysics. Book 1. Theoretical Biophysics]. Moscow, Vysshaya shkola Publ., 1987. 319 p.

8. Rubin A.B. Biofizika. Kn.2. Biofizika kletochnykh protsessov [Biophysics. Book 2. Biophysics of Cellular Processes]. Moscow, Vysshaya shkola Publ., 1987. 303 p.

9. Teylor E., Uiler Dzh. Fizika prostranstvavremeni [Physics of Space-Time]. Moscow, Mir Publ., 1971. 320 p.

10. Korablev G.A. Spatial-Energy Principles of Complex Structures Formation. Monograph. Brill Academic Publishers and VSP, Netherlands, 2005. $426 \mathrm{p}$.

\section{ENTROPIC NOMOGRAM}

\section{Korablev Grigoriy Andreevich}

Doctor of Chemical Sciences, Professor,

Head of Department of Physics,

Izhevsk State Agricultural Academy

korablevga@mail.ru

Studencheskaya St., 11, 426069 Izhevsk, Russian Federation

\section{Petrova Natalya Grigoryevna}

Specialist-Expert,

Department of Information Security and Communications,

Ministry of Informatization and Communications, Udmurt Republic

biakaa@mail.ru

Vadima Sivkova St., 186, 426057 Izhevsk, Russian Federation

\section{Kodolov Vladimir Ivanovich}

Doctor of Chemical Sciences, Professor,

Head of Department of Chemistry and Chemical Technology,

Izhevsk State Technical University

kodol@istu.ru

Studencheskaya St., 7, 426000 Izhevsk, Russian Federation

\section{Zaikov Gennady Efremovich}

Doctor of Chemical Sciences, Professor,

Head of Department of Biological and Chemical Physics of Polymers, Institute of Biochemical Physics named after N. M. Emanuel, RAS

chembio@sky.chph.ras.ru

Kosygina St., 4, 119334 Moscow, Russian Federation 


\title{
Korablev Roman Grigoryevich
}

Postgraduate Student, Izhevsk State Agricultural Academy

korablevga@mail.ru

Studencheskaya St., 11, 426069 Izhevsk, Russian Federation

\section{Osipov Anatoliy Konstantinovich}

Doctor of Economic Sciences, Professor,

Head of Department of Management and Law,

Izhevsk State Agricultural Academy

korablevga@mail.ru

Studencheskaya St., 11, 426069 Izhevsk, Russian Federation

\begin{abstract}
The concept of entropy spatial-energy interactions is used to reflect the similar views on the statistical thermodynamics of entropy.

The resulting nomogram is obtained for estimating the entropy of various processes. The authors discuss the variety of manifestations of entropy, including the biochemical processes in the economy.

In living systems the entropy increase is offset by the negative entropy, which is formed through interaction with the external environment. Thus, a living system is an open system. And businesses can no longer be isolated systems without exchange process and the relationship with the external environment. The role of the external system reducing the increase in business entropy must perform, for example, relevant state and public structures, functionally separated from the business. Perhaps, inevitable process in this direction can be de-monopolization of the largest economic structures, carried out "on top" in an evolutionary way.

In thermodynamics it is considered that the uncontrolled growth of entropy leads to the termination of any macroeconomic systems, that is, to their death. Therefore, the main task is to search for the methods of reducing the uncontrolled growth of entropy in big business. In addition, these critical numbers of entropy relate mainly to big business. A simple reduction of the number of its employees may not give the real result of a decrease in entropy. So, reduction of the number of employees by $10 \%$ would decrease the entropy of only $0.6 \%$ and this despite the general negative effects of unemployment, which inevitably accompanies such a process.

Therefore, such sermonettes not controlled either by the state or society are aimed to reduce the entropy of the business in a more real way of demonopolization without optimization (that is, without reducing the total number of employees).

Now the scientific world is puzzled by the intensification of technological processes on the basis of energy-saving technology, for example. This technique P-parameter can also be used in this promising direction.
\end{abstract} business.

Key words: entropy, nomogram, spatial-energy parameter, carbonization, diffusion, 